\title{
Mitteilung \\ uber die Berechnung der Lage der eutektischen Punkte und der Löslichkeitsgrenzen bei Systemen mit Eisen.
}

Von KarL Daeves.

In grundlegenden Untersuchungen hat G. TAMManN ${ }^{1}$ ) entdeckt, daß die Einwirkungsgrenzen chemischer Agenzien auf Mischkristallreihen bis zu bestimmten, scharf zu ermittelnden Gelialten reichen, die, als Bruchteile aller vorhandenen Atome ausgedrückt, ganzzahlige Vielfache ron $1 / 8$ sind.

Im Folgenden soll eine vorläufige Mitteilung über ein ähnliches, die in der Metallographie so wichtigen Grenzen der Löslichkeit und die Lage der eutektischen Punkte regelndes Gesetz gemacht werden.

Bei Untersuchungen über die Einwirkungsgrenzen anderer Reihen kam der Verfasser zu der überraschenden Entdeckung, daß sich in einer ganzen Reihe ron Fällen die eutektischen Punkte, sowie die Grenzen. der Löslichkeit ebenfalls als n/8 Molbrüche ausdrücken lassen. Voraussetzung ist nur, daB die Elemente und Verbindungen mit dem ihnen in den betreffenden Systemen zukommenden Molekulargewicht eingesetzt werden.

Als Beispiele seien angeführt:

1. Setzt man im System $\mathrm{Fe}-\mathrm{C}$ das $\mathrm{Fe}$ als dreiatomig, das $\mathrm{C}$ als in $\mathrm{Fe}_{3} \mathrm{C}$ enthalten, ein, und bildet die Molenbrüche zwischen $\mathrm{Fe}_{3}$ und $\mathrm{Fe}_{3} \mathrm{C}$, so ist

$$
\begin{aligned}
& 1 / 8 \mathrm{Mol}=13,3 \% \mathrm{Fe}_{3} \mathrm{C}=0,89 \% \mathrm{C}, \\
& 2 / 8 \mathrm{Mol}=26,2 \% \mathrm{Fe}_{3} \mathrm{C}=1,75 \% \mathrm{C}, \\
& 5 / 8 \mathrm{Mol}=64,2 \% \mathrm{Fe}_{3} \mathrm{C}=4,29 \% \mathrm{C}, \\
& 8 / 8 \mathrm{Mol}=100 \% \mathrm{Fe}_{3} \mathrm{C}=6,7 \% \mathrm{C} .
\end{aligned}
$$

Im thermischen Diagramm sind diese Punkte als Perlitpunkt, Grenze der Löslichkeit für C, eutektischer Punkt und als Verbindung $\mathrm{Fe}_{\mathbf{3}} \mathrm{C}$ charakteristisch.

1) Z. anorg. u. allg. Chem. 107 (1919), S. 1 ti: 
2. Im System $\mathrm{Fe}-\mathrm{Ti}$ wird $\mathrm{Fe}$ als $\mathrm{Fe}_{3}$, $\mathrm{Ti}$ als in $\mathrm{Fe}_{3} \mathrm{Ti}$ enthaltén eingeführt und man erbält

$$
\begin{aligned}
& 2 / 8 \mathrm{Mol}=30,0 \% \mathrm{Fe}_{3} \mathrm{Ti}=6,7 \% \mathrm{Ti}, \\
& 5 / 8 \mathrm{Mol}=68,1 \% \mathrm{Fe}_{3} \mathrm{Ti}=15,2 \% \mathrm{Ti} .
\end{aligned}
$$

Die erste $\mathbb{K}_{\text {Konzentration stimmt gut mit dem von LaMoRT }}{ }^{1}$ ) gefundenen Wert überein, die zweite ist $2 \%$ höher als der von ihm für den eutektischen Punkt gefundenen Wert.

3. Im System $\mathrm{Fe}-\mathrm{P}$ wird $\mathrm{Fe}$ als $\mathrm{Fe}_{3}, \mathrm{P}$ als $\mathrm{Fe}_{3} \mathrm{P}$ eingesetzt und man findet

$$
\begin{aligned}
& 1 / 8 \mathrm{Mol}=10,75 \% \mathrm{Fe}_{3} \mathrm{P}=1,68 \% \mathrm{P}, \\
& 5 / 8 \mathrm{Mol}=66,4 \% \mathrm{Fe}_{3} \mathrm{P}=10,35 \% \mathrm{P} .
\end{aligned}
$$

Beide Werte stimmen gut mit den von Gercke und andern gefundenen Werten für Löslichkeitsgrenze und eutektischen Punkt überein.

4. Im System $\mathrm{Fe}-\mathrm{As}$, betrachtet als System $\mathrm{Fe}_{2} \mathrm{As}-\mathrm{Fe}_{2}$ ergibt sich

$$
\begin{aligned}
& 1 / 8 \mathrm{Mol}=19,3 \% \mathrm{Fe}_{2} \mathrm{As}=7,25 \% \mathrm{As}, \\
& 5 / 8 \mathrm{Mol}=73,7 \% \mathrm{Fe}_{2} \mathrm{As}=29,6 \% \mathrm{As} .
\end{aligned}
$$

Beide Werte stimmen mit den für die Löslichkeitsgrenze und den eutektischen Punkt bestimmten gut überein.

5. Während aber beim System $\mathrm{Fe}-\mathrm{B}$ das naheliegende Einsetzen der Komponenten $\mathrm{Fe}_{5} \mathrm{~B}_{2}$ und $\mathrm{Fe}_{5}$ die Werte ergeben würde

$$
\begin{aligned}
& 1 / 8 \mathrm{Mol}=13,4 \% \quad \mathrm{Fe}_{5} \mathrm{~B}_{2}=0,98 \% \mathrm{~B}, \\
& 5 / 8 \mathrm{Mol}=64,4 \% \mathrm{Fe}_{5} \mathrm{~B}_{2}=4,7 \% \mathrm{~B},
\end{aligned}
$$

die schlecht mit den thermisch gefundenen Angaben Hannessans ${ }^{2}$ ) übereinstimmen, ergibt eine Betrachtung als System $\mathrm{Fe}_{3} \mathrm{~B}-\mathrm{Fe}_{3}$

$$
\begin{aligned}
& 1 / 8 \mathrm{Mol}=13,2 \% \mathrm{Fe}_{3} \mathrm{~B}=0,82 \% \mathrm{~B}, \\
& \% \mathrm{Mol}=64,0 \% \mathrm{Fe}_{3} \mathrm{~B}=3,95 \% \mathrm{~B},
\end{aligned}
$$

Zahlen, die mit den von HANNESSEN erhaltenen übereinstimmen. Das System wäre also daraufhin nachzuprüfen, ob nicht an Stelle der Verbindung $\mathrm{Fe}_{5} \mathrm{~B}_{2}$ die dem $\mathrm{Fe}_{3} \mathrm{C}$ analoge $\mathrm{Fe}_{3} \mathrm{~B}$ auftritt.

Die Beispiele lieben sich vermehren, jedoch sei darauf hingewiesen, daB bei vielen Systemen, besonders solchen, die keine Verbindung bilden, die Verhältnisse nicht so einfach liegen, zumal man dann betreffs des Molekulargewichts der Komponenten auf Vermutungen angewiesen ist. Die Untersuchungen werden fortgesetzt.

1) Ferrum 1914, S. 225.

2) Z. anorg. Chem. 89, 257. 
Es läBt sich aber schon jetzt das Gesetz festlegen: Die in den Binärsystemen des Eisens auftretenden charakteristischen Punkte der Löslichkeitsgrenzen und die eutektischen Pankte lassen sich in vielen Fällen durch n/8 Mol Brüche ausdrücken. Voraussetzung ist, da $B$ die Elemente mit den ihnen in den betreffenden System zukommenden Molekulargewicht eingesetzt werden.

Es ist anzunehmen, daB das Gesetz noch viel allgemeiner gilt, als oben ausgedrückt und daB sich aus ihm weitere GesetzmäBigkeiten ergeben werden (z. B. scheint fast immer bei $5 / 8 \mathrm{Mol}$ ein Eutektikum aufzutreten). Jedenfalls ermöglicht das Gesetz eine Kontrolle der durch thermische Analyse gefundenen Systeme, es wird vielleicht in vervollkommneter Form die Vorausberechnung ron Systemdiagrammen gestatten und uns wertrolle Aufklärung über die Molekularstruktur der für die Technik so wichtigen Legierungen geben.

Bismarckhütte $\mathbf{O} / \mathbf{S}$, Versuchsanstalt der Bismarckhütte.

Bei der Redaktion eingegangen am 26. November 1920 . 